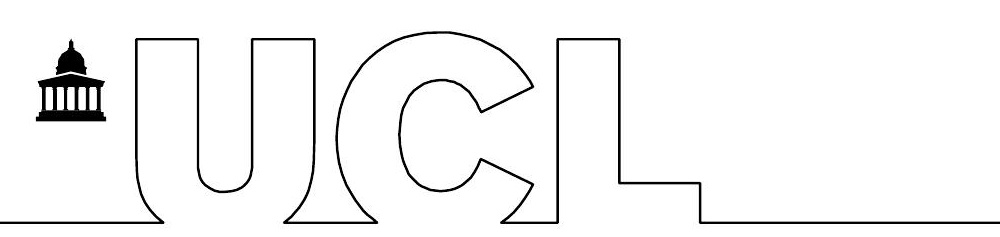

Ferguson, SG; Brown, J; Frandsen, M; West, R; (2015) Associations between use of pharmacological aids in a smoking cessation attempt and subsequent quitting activity: a population study. Addiction , 110 (3) 513 518. 10.1111/add.12795. Downloaded from UCL Discovery: http://discovery.ucl.ac.uk/1457914

\title{
ARTICLE
}

\section{Associations between use of pharmacological aids in a smoking cessation attempt and subsequent quitting activity: a population study}

\author{
Stuart G. Ferguson, Ph. $D^{1}$, Jamie Brown, Ph. ${ }^{2}$, Mai Frandsen Ph. $D^{1}$, Robert West, Ph. $D^{2}$ \\ ${ }^{1}$ School of Medicine, University of Tasmania, Hobart, Australia \\ ${ }^{2}$ Cancer Research UK Health Behaviour Research Centre, University College London, WC1E \\ 6BT, UK \\ Corresponding Author: Dr Stuart Ferguson, School of Medicine, University of Tasmania, Private \\ Bag 68, Hobart, 7001, AUSTRALIA; email: Stuart.Ferguson@utas.edu.au; Ph: +61 36226 4295; \\ Fax: +61362262870 .
}

\section{Declaration of Interests}

SGF has consulted for GlaxoSmithKline Consumer Healthcare (GSKCH) on matters relating to smoking cessation and has received researcher-initiated project grant funding from Pfizer (through the GRAND initiative). JB has received an unrestricted research grant from Pfizer. RW undertakes research and consultancy for, and has received travel funds and hospitality from, companies that develop and manufacture smoking cessation medications. He has a share in a patent for a novel nicotine delivery device. This study is funded by the UK Department of Health, Cancer Research UK, Pfizer, GSKCH and J\&J, who had no involvement in the design of the study, collection, analysis or interpretation of the data, the writing of the report, or the decision to submit the paper for publication. JB's post is funded by a fellowship from the UK Society for the Study of Addiction; RW is funded by Cancer Research UK but work independently from the funders listed; MF is supported through a fellowship from Cancer Council Tasmania (Australia).

\begin{abstract}
Background and Aims: Modelling the population impact and cost-effectiveness of smoking cessation aids is limited by lack of knowledge about how the use of aids changes across quit attempts. Here we test whether the quit method used in a previous attempt influences (i) future decisions to quit and/or (ii) treatment/s used during subsequent attempts.
\end{abstract}

Design and Setting: Data came from the Smoking Toolkit Study, a United Kingdom national household survey.

Participants and Measures: Smokers $(n=5489)$ who completed a baseline and 6-month follow-up questionnaire. Respondents were asked what treatment/s, grouped as: prescription medication/s [bupropion, varenicline or nicotine replacement therapy (NRT)], over-the-counter NRT or unaided that they had used in their most recent quit attempt (at baseline), and any use of treatment/s for a quit attempt in the last 3 months at follow-up. 
Results: Smokers who had tried to quit at baseline were more likely to report having tried to quit again prior to follow-up [all odds ratios $\geq 2.19$ relative to no attempt at baseline, $P<0.001$ ].

Smokers who tried to quit using pharmacological aids were more likely to try to quit again at followup (all ORs $\geq 2.19$ relative to no attempt at baseline, $P<0.001$ ). Smokers tended to re-try aids used in baseline attempts in future attempts (all ORs $\geq 1.48$ relative to no attempt at baseline, $\mathrm{P}<0.01)$.

Conclusions: Smokers who have tried to quit in the past year are more likely to try to quit again within 6 months regardless of whether they used a pharmacological aid, and they are more likely to re-try aids they had used previously.

Keywords: Smoking cessation, quitting behaviour, cessation medications

\section{Introduction}

When modelling the impact of smoking cessation interventions, it is assumed that smokers who do not succeed in quitting following the intervention have a similar chance of quitting subsequently as the general population of smokers; that is they quit at the 'background quit rate' $(1,2,3)$. However, this assumption is unlikely to be correct. For example, it has been shown that smokers who try and fail to stop are more likely to try again in subsequent months when followed up (4). What is not known is how far this applies to specific methods of stopping, such as use of pharmacological aids. Neither is it known whether smokers using a given method on one occasion are more or less likely to use the same method on subsequent occasions. This paper provides an initial estimate of these parameters from a large national sample of smokers in England.

The issue of whether smokers who try to stop using different methods are more or less likely to make a subsequent quit attempt has important implications for modelling the longer-term impact of those interventions. For example, if failing to stop smoking after using nicotine replacement therapy (NRT) bought over the counter (OTC) decreases likelihood of making a subsequent quit attempt, this would negatively impact on population quitting rates using this method. This would need to be taken into account in any recommendations. On the other hand, if users of OTC NRT were more likely to try to stop subsequently if they failed on a given occasion relative to those using other methods, this would increase the population impact. Similar, considerations would apply for other quitting methods.

The association between the use of different quitting methods on use of the same versus other methods is similarly important in terms of longer-term population impact. For example, if smokers who use a prescription medicine to stop on one occasion are more likely to use the same method again if they fail, this could influence their longer-term chance of quitting successfully. This would need to be taken into account in cost-effectiveness modelling. Knowledge of the use of quit methods over time is also of interest to clinicians involved in counselling patients to quit. One potential detrimental effect of a failed quit attempt is that smokers may attribute failure to the medication or treatment used. This raises the concern that a smoker, who fails during an aided quit attempt, may be less motivated to quit or use the same, or other treatments, in future quit attempts, thus reducing their chances of successfully quitting. If failure negatively impacts on a smoker's likelihood of using a particular treatment again, this would suggest that we need to exercise caution 
when encouraging the use of any treatment, perhaps only doing so in cases where smokers are highly-motivated to make a serious quit attempt, and, in the case of pharmacotherapy in particular, only when patients have been educated about the importance of compliance and adherence.

To our knowledge, there is no direct evidence on these issues in the literature and behavioural theory suggests several factors that would influence behaviour in different directions. For example, it is possible that smokers who fail to stop using a given method that has made it subjectively easier (e.g., by reducing craving and withdrawal severity), even though they relapsed, would be encouraged to make another attempt and to use the same method again. However, it is also possible that failure to quit with a method that they had expected to be effective could discourage future quitting attempts and/or lead to switching to another method. In the absence of a steer from theory or previous studies the present study has to be considered exploratory.

The present study made use of an on-going surveillance programme in England called the Smoking Toolkit Study (STS) $(5,6)$. This consists of a series of monthly household surveys of nationally representative samples aged 16+. Questions focus on smoking status, smoking patterns and quitting activity. Each month involves a new sample. The study has been in operation since November 2006. A number of the monthly surveys have been supplemented by a postal questionnaire 6 months later. This provides an opportunity to examine prospective associations between quitting activities over two different time points. There are many possible approaches to assessing associations between quitting methods and subsequent quit attempts and quitting methods, each with strengths and limitations. For this initial study, we chose the simplest approach which was to assess the method used in the most recent attempt made in the past 12 months at baseline and assess associations between this and 1) whether a subsequent quit attempt was recorded at a 6-month follow up (within the last 3 months), and 2) the method used in any such quit attempt. We did not assess the more complex modelling of conditional probabilities between successive quit attempts where more than one quit attempt was made prior to baseline or between the baseline and follow-up would require a larger sample and additional longitudinal data not available in the STS. In order to rule out associations arising from sources of common variance such as age, gender, social grade and nicotine dependence, we undertook all analyses with and without adjustment for these variables.

In England, unaided quitting, OTC NRT, and use of a prescription medication are the most commonly used methods $(7,8)$ and therefore STS users of these methods provided sample sizes sufficient to obtain parameter estimates with acceptable confidence intervals, given the accumulated sample size of the study. Use of behavioural support is common relative to other countries but still would not provide an adequate sample size for this study, so was excluded. Thus the research question addressed by this study was: after adjusting for important sources of common variance, how far is method used to quit in the most recent quit attempt made within the past 12 months (in terms of unaided cessation, use of OTC NRT or use of a prescription medication) predictive of a) odds of a quit attempt in the next 6 months, and b) quitting method among those making at least one attempt.

\section{Methods \\ Overview}


As noted above, data were drawn from the STS. Participants are recruited via random sampling of grouped output areas (300 households to each area) stratified by ACORN characteristics (9) and region. Trained interviewers randomly select households within these areas and conduct face-toface computer assisted interviews with one household member ( $\geq 16$ years old) per residence until quotas are reached. For some waves of the STS, subject to funding constraints, 6 months after this initial baseline survey, current smokers and recent quitters, who had consented to being recontacted, were posted a follow-up questionnaire. Full details of the methodology and sample representativeness have been described elsewhere $(5,6)$.

\section{Sample}

The present sample was drawn from current smokers who completed the baseline survey prior to April 2012 (when follow ups were suspended) and were successfully followed up. Respondents were classified as current smokers at baseline and at follow up if they reported smoking cigarettes or tobacco products (e.g., hand rolled, cigar, or pipe) every day or occasionally. Of a baseline sample of 25,621 smokers, 5,489 were followed up.

\section{Measures}

Quit attempts in the preceding 12 months were assessed by asking: "How many serious attempts to stop smoking have you made in the last 12 months? By serious attempt I mean you decided that you would try to make sure you never smoked again. Please include any attempt that you are currently making and please include any successful attempt made within the last year". Among those reporting at least one attempt, the use of smoking cessation aids was assessed for the most recent quit attempt by asking "Which, if any, of the following did you try to help you stop smoking during the most recent serious quit attempt? " and respondents could choose between response categories; for analysis, we collapsed responses into three groups: i) medication (NRT, bupropion or varenicline) provided or prescribed by health-care professional (MED-RX); ii) NRT bought OTC (OTC NRT); and (iii) unaided (i.e., had not used any of NRT bought, a prescription stop-smoking medication or face-to-face behavioural support). If a smoker reported using OTC NRT and MED$\mathrm{RX}$ in their most recent quit attempt, they were coded as using MED-RX. [This only occurred in a small number $(\mathrm{n}=28)$ of cases.] Smokers were also asked how long ago the quit attempt started. Note that, as they were all smokers, the quit attempt had not succeeded.

Demographic characteristics (age, gender and social grade) and cigarettes per day (CPD) were also assessed at baseline. Social grade was based on the household's main income earner and calculated according to the British National Readership Survey classification system and dichotomised into two categories: $\mathrm{ABC} 1$ which includes managerial, professional and intermediate occupations; and C2DE which includes small employers and own account workers, lower supervisory and technical occupations, and semi-routine and routine occupations, never workers, and long-term unemployed.

Participants were posted a follow-up questionnaire 6 months after the initial baseline survey. The follow-up questionnaire asked similar questions to the baseline survey about quit attempts over the past year. To avoid possible overlap between these quit attempts and those recorded at baseline we restricted consideration to attempts made in the past 3 months. If more than one quit attempt was reported within this time period $(n=178)$, or if more than one method was reported within a single attempt, we coded the highest intensity method used (i.e., a respondent was classified as 
having used MED-RX if they used this and OTC NRT, OTC NRT if they reported any OTC NRT but no MED-RX, and as unaided if they reported no use of OTC NRT nor MED-RX). Ethical approval was granted by the University College of London Research Ethics Committee.

\section{Analytic plan}

Contingency tables showing the proportions making a quit attempt using different methods at follow-up as a function methods of quitting at baseline were prepared. Then simple and multiple logistic regressions were undertaken to assess a) the odds of making a quit attempt and b) the odds of making a quit attempt using a particular method as a function of method used at baseline. In all cases, not having made a quit attempt at baseline was used as the common reference category. The multiple regression models controlled for baseline measures of age, gender, social grade (ABC1 vs. C2DE), CPD and year of survey.

\section{Results}

\section{Baseline characteristics}

The study sample comprised the 5,489 individuals who returned the follow-up survey; $21.4 \%$ of the 25,621 eligible current smokers surveyed at baseline. Compared with those lost to follow-up, respondents in the evaluable sample were older, more likely to be female, smoked a greater number of CPD and showed a different pattern of quitting during the year prior to the baseline survey (Table 1). The proportion from a routine and manual occupation was similar between the two groups.

At baseline, the majority (68.9\%) of respondents reported not having made a quit attempt in the previous 12 months. Among those who had made a quit attempt $(n=1,707)$, a third $(33.3 \%)$ reported using OTC NRT during their most recent quit attempt and $20.3 \%$ reported using prescription medications, and approximately half (46.3\%) of respondents reported that their most recent quit attempt was unassisted.

[Insert Table 1 around here]

\section{Quitting behaviour at 6-month follow-up}

Just over a fifth $(21.7 \% ; n=1,191)$ of respondents reported having made a quit attempt in the 3 months up to the 6-month follow-up survey. Table 2 shows the proportions and odds of making a quit attempt at follow-up, based on quitting behaviour at baseline. Compared with smokers who reported not having made a quit attempt in the last year at baseline, respondents who reported that they had tried to quit at baseline had two to three times greater odds of reporting having attempted to quit at follow-up (Table 2). Over a third of respondents who reported having tried to quit unassisted during their most recent quit attempt at baseline reported having made a subsequent quit attempt at the follow-up survey, compared with just $15.8 \%$ of baseline responders who reported not having made a quit attempt in the last year at baseline. Similarly, smokers who tried to quit using smoking cessation medications at baseline were more likely to report having tried to quit again at follow-up (Table 2).

[Insert Table 2 around here] 
Table 3 shows the association between baseline quitting behaviour and subsequent behaviour at follow-up among those participants who reported making quit attempt at the follow-up survey $(n=1,191)$. Compared with those who had not attempted to quit in the last year at baseline, respondents who indicated that their most recent attempt to quit at baseline had been unaided were more likely to also make a subsequent unaided attempt (67.9\% versus $76.2 \%$; Table 3 ). Conversely, those who used medications at baseline - either OTC or on prescription - had significantly lower odds of attempting to quit unaided at follow-up (Table 3). Similarly, the use of OTC NRT or a prescription medication with brief advice at baseline was associated with increased odds of using these treatments again at follow-up.

[Insert Table 3 around here]

\section{Discussion}

Our results indicate that there are increased odds of making a quit attempt if one has been made in the recent past and this is not substantially affected by whether or not the previous attempt involved use of pharmacotherapy. They also show that the odds are increased for using the same method as one used before, whether it be no pharmacotherapy, OTC NRT or prescription medication.

The results suggest that modelling the impact of use of different pharmacological aids to cessation should factor in an adjustment to background quitting rates. In order to know how much to raise such rates one would have to have much longer follow-up and would also have to consider the chances of success of subsequent quit attempts. This is an important area for future research.

From a clinical and policy perspective, it would seem undesirable that smokers using methods of quitting that for them have not proved successful, should show a tendency to use the same method again. It would seem reasonable to suggest to smokers who have not succeeded with a lower level of support and help that they should try with a higher level of support the next time. If smokers have not succeeded with a higher level of support, e.g. a prescription medication, it would seem reasonable to examine what more could be done to improve their chances of success next time. This might involve using behavioural support, adding additional self-help methods, or using whatever method they are using more intensively (10). For example, if they had used a single form NRT, they could be advised to use a dual form (transdermal patch plus a faster acting form); or they could be advised to use higher dose products, use them more often or for longer (11).

There is little information on what happens when smokers use the same method of stopping as previously but a recent placebo controlled trial found that smokers who had previously used varenicline in a quit attempt but resumed smoking, benefitted from using the same medication again relative to placebo (12). The effect size was similar to what is observed with smokers who have not used this medication before, indicating that in this case there is merit in using the same method again (13).

The study had several limitations. 1) The follow up rate was low and the sample followed up was different from that not followed up in relation to a number of factors. However, the differences were 
small and unlikely to have made a substantial difference to the effect size estimates. In any case, these differences (age, gender, CPD, and number of quit attempts made in the previous 12 months at baseline) were controlled for in our analyses. 2) Failed quit attempts are often forgotten $(14,15)$ and it is possible that this may have influenced the size of the associations observed. If forgetting of all types of quit attempt was at the same rate this would not substantially effect the findings but if unaided quit attempts are forgotten more quickly than ones using pharmacotherapy (14) this could bias the results against consistency in use of unaided quitting. 3) The study only looked at quitting activity over a period of up to 18 months (12 months prior to the baseline and then leading up to the 6-month follow up). Longer-term studies are required. 4) As an initial investigation into the issue, this study simplified the task by looking at the most recent quit attempts leading up to the baseline surveys. Ideally one would undertake a more comprehensive analysis of the conditional probabilities of quitting using different methods. However, this is hampered by the need to adjust for background variables and the need to ensure that what are reported as different quit attempts really are different, rather than continuations of an existing quit attempt. This requires development of an improved methodology for assessing quit attempts. 5) The study did not examine consistency in use of specific pharmacotherapies nor other features of the quit attempt (e.g. abrupt versus gradual, planned versus unplanned, with or without behavioural support). These are important areas for future study.

Despite these limitations, this study provides evidence that whether or not smokers use pharmacological aids to a quit attempt does not seem to alter their likelihood of making another quit attempt in the following months and that smokers show some consistency in their choice of quitting method. It would be worth considering how these finding can be incorporated into costimpact models of different smoking cessation interventions.

\section{Acknowledgements}

We wish to thank Isabelle Morris and Dr John Cianchi for providing comments on drafts. 


\section{References}

1. Fiscella K, Franks P. Cost-effectiveness of the transdermal nicotine patch as an adjunct to physician's smoking cessation counselling. Journal of the American Medical Association 1996;275:1247-51. doi:10.1001/jama.275.16.1247.

2. Stapleton JA, West R. A direct method and ICER tables for the estimation of the costeffectiveness of smoking cessation interventions in general populations: application to a new cytisine trial and other examples. Nicotine \& Tobacco Research 2012;14(4):463-71.

3. Brown J, Kotz D, Michie S, Stapleton J, Walmsley M, West R. How effective and costeffective was the national mass media smoking cessation campaign 'Stoptober'? Drug and Alcohol Dependence 2014;135:52-8.

4. Partos TR, Borland $\mathrm{R}$, Yong $\mathrm{H}-\mathrm{H}$, Hyland $\mathrm{A}$, Cummings $\mathrm{KM}$. The quitting rollercoaster: how recent quitting history affects future cessation outcomes (data from the International Tobacco Control 4-country cohort study). Nicotine \& Tobacco Research. 2013;15:1578-87. doi: $10.1093 /$ ntr/ntt025.

5. Fidler JA, Shahab L, West O, Jarvis MJ, McEwen A, Stapleton JA, et al. The smoking toolkit study: a national study of smoking and smoking cessation in England. BMC Public Health 2011;11:479-487.

6. Fidler JA, Ferguson SG, Brown J, Stapleton J, West R. How does rate of smoking cessation vary by age, gender and social grade? Findings from a population survey in England. Addiction 2013;108:1680-1685. doi:10.1111/add.12241.

7. Kotz D, Fidler J, West R. Factors associated with the use of aids to cessation in English smokers. Addiction 2009;104:1403-1410. doi: 10.1111/j.1360-0443.2009.02639.x.

8. Shiffman S, Brockwell SE, Pillitteri JL, Gitchell JG. Use of smoking-cessation treatments in the United States. Am J Prev Med 2008;34(2):102-11. doi: 10.1016/j.amepre.2007.09.033.

9. CACI. Acorn: Understanding consumers and communities [updated: 2013, cited 2013 May 23]. Available from http://acorn.caci.co.uk/

10. Fiore MC, Jaen CR, Baker TB, Bailey WC, Benowitz NL, Curry S et al. Treating tobacco use and dependence: 2008 Update. Clinical practice guideline. 2008. U.S. Department of Health and Human Services, Rockville.

11. Stead LF, Perera R, Bullen C, Mant D, Hartmann-Boyce J, Cahill K, Lancaster T. Nicotine replacement therapy for smoking cessation. Cochrane Database of Systematic Reviews 2012, Issue 11. Art. No.: CD000146. DOI: 10.1002/14651858.CD000146.pub4.

12. Gonzales D, Hajek P, Pliamm L, Nackaerts K, Tseng LJ, McRae TD, Treadow J. Retreatment with varenicline for smoking cessation in smokers who have previously taken varenicline: A randomized, placebo-controlled trial. Clin Pharmacol Ther 2014; [Epub ahead of print]. doi: 10.1038/clpt.2014.124.

13. Cupertino A, Wick JA, Richter KP, Mussulman L, Nazir N, Ellerbeck EF. The impact of repeated cycles of pharmacotherapy on smoking cessation: A longitudinal cohort study. Arch Intern Med. 2009;169(20):1928-30.

14. Borland R, Partos TR, Cummings KM. Systematic biases in cross-sectional community studies may underestimate the effectiveness of stop-smoking medications. Nicotine \& Tobacco Research 2012;14(12):1483-7. doi: 10.1093/ntr/nts002.

15. Berg CJ, An LC, Kirch M, Guo H, Thomas JL, Patten CA, et al. Failure to report attempts to quit smoking. Addictive Behaviors 2010;35(10):900-4. 
Table 1: Comparison of baseline characteristics of those in the evaluable study sample $(n=5,489)$ versus those lost to 6 -month follow-up $(n=20,132)$.

\begin{tabular}{lll}
\hline & $\begin{array}{l}\text { Study sample } \\
(\mathrm{n}=5,489)\end{array}$ & $\begin{array}{l}\text { Lost to follow-up } \\
(\mathrm{n}=20,132)\end{array}$ \\
\hline Mean age (SD) & $47.1(15.5)$ & $40.9(16.4)^{*}$ \\
\% Female (n) & $56.3(3,089)$ & $50.1(10,086)^{\star}$ \\
\% C2DE Social grade (n) & $68.2(3,744)$ & $68.2(13,725)$ \\
Mean CPD (SD) & $14.2(8.8)$ & $13.1(8.5)^{\star}$ \\
Method used in most recent quit attempt & & \\
$\quad$ No quit attempt (in last 12 months) & $68.9(3,782)$ & $66.3(13,340)^{\star}$ \\
$\quad$ Unaided & $14.4(791)$ & $17.6(3,551)$ \\
$\quad$ OTC NRT & $10.4(569)$ & $10.8(2,165)$ \\
$\quad$ Medication on prescription \& brief advice & $6.3(347)$ & $5.3(1,076)$ \\
\hline
\end{tabular}

Notes: OTC NRT = Over-the-counter nicotine replacement therapy. Differences between the two groups were assessed with $x^{2}$ tests and one-way ANOVAs for categorical and continuous variables respectively. ${ }^{*} p<.001$. 
Table 2: The association between quitting behaviour at baseline and reporting having made a quit attempt in the last 3 months at 6 months follow-up.

Quit attempts recorded at baseline
$\%$ reporting having made a quit attempt

in last 3 months (at follow-up)

No attempts to quit in previous 12 months

(Reference group; $n=3,782$ )

Unaided $(n=791)$

OTC NRT $(n=569)$

Medication on prescription \& brief advice $(n=347) \quad 28.2$ (98)

$2.98^{* * *}$

$3.30^{* * *}$

$2.19^{\star \star *}$

Odds Ratio

95\% Confidence Interval

Notes: OTC NRT = Over-the-counter nicotine replacement therapy. The models are adjusted for all variables listed in Table 1 and the year of the survey. ${ }^{* * *} \mathrm{p}<.001$. 
Table 3: The association between quitting behaviour at baseline and treatment use among those who reported attempting to quit in the last 3 months at the 6 months follow-up $(n=1,191)$.

Treatment used during last 3 months prior to 6-months follow-up

Quit attempts recorded at baseline

\begin{tabular}{llllll}
\hline $\begin{array}{l}\text { \% Unaided } \\
(\mathrm{n})\end{array}$ & OR & \% OTC NRT & OR & \% Med Rx \& & OR \\
& $(95 \% \mathrm{Cl})$ & $(\mathrm{n})$ & $(95 \% \mathrm{Cl})$ & brief advice $(\mathrm{n})$ & $(95 \% \mathrm{Cl})$
\end{tabular}

\begin{tabular}{|c|c|c|c|c|c|c|}
\hline $\begin{array}{l}\text { No attempts to quit in previous } 12 \\
\text { months (Reference group; } n=599 \text { ) }\end{array}$ & $67.9(407)$ & - & $20.0(120)$ & - & $12.0(72)$ & - \\
\hline Unaided $(n=281)$ & $76.2(214)$ & $\begin{array}{l}1.48^{*} \\
(1.07 \text { to } 2.06)\end{array}$ & $16.7(47)$ & $\begin{array}{l}0.78 \\
(0.54 \text { to } 1.14)\end{array}$ & $7.1(20)$ & $\begin{array}{l}0.61 \\
(0.36 \text { to } 1.02)\end{array}$ \\
\hline OTC NRT $(n=213)$ & $45.5(97)$ & $\begin{array}{l}0.40^{\star * \star} \\
(0.29 \text { to } 0.55)\end{array}$ & $39.4(84)$ & $\begin{array}{l}2.57^{\star \star \star} \\
(1.83 \text { to } 3.63)\end{array}$ & $15.0(32)$ & $\begin{array}{l}1.31 \\
(0.83 \text { to } 2.06)\end{array}$ \\
\hline $\begin{array}{l}\text { Medication on prescription \& brief } \\
\text { advice }(n=98)\end{array}$ & $42.9(42)$ & $\begin{array}{l}0.38^{* * *} \\
(0.25 \text { to } 0.59)\end{array}$ & $30.6(30)$ & $\begin{array}{l}1.69^{*} \\
(1.05 \text { to } 2.73)\end{array}$ & $26.5(26)$ & $\begin{array}{l}2.47^{\star * *} \\
(1.47 \text { to } 4.14)\end{array}$ \\
\hline
\end{tabular}

Notes: OTC NRT $=$ Over-the-counter nicotine replacement therapy. Med $\mathrm{Rx}=$ medication on prescription. $\mathrm{Cl}=\mathrm{Confidence}$ Interval. All models adjusted for all variables listed in Table 1 and the year of the survey. ${ }^{*} p<.05,{ }^{* \star *} p<.001$. 